\title{
Modelling the spin pulse profile of the isolated neutron star RX J0720.4-3125 observed with XMM-Newton`
}

\author{
M. Cropper ${ }^{1}$, S. Zane ${ }^{1}$, G. Ramsay ${ }^{1}$, F. Haberl ${ }^{2}$, and C. Motch ${ }^{3}$ \\ 1 Mullard Space Science Laboratory, University College London, Holmbury St. Mary, Dorking, Surrey, \\ RH5 6NT, UK \\ 2 Max-Planck-Institut für Extraterrestrische Physik, Giessenbachstrasse, 85748 Garching, Germany \\ 3 Observatoire Astronomique, CNRS UMR 7550, 11 rue de l'Université, 67000 Strasbourg, France
}

Received 28 September 2000 / Accepted 31 October 2000

\begin{abstract}
We model the spin pulse intensity and hardness ratio profiles of the isolated neutron star RX J0720.43125 using XMM-Newton data. The observed variation is approximately sinusoidal with a peak-to-peak amplitude of $15 \%$, and the hardness ratio is softest slightly before flux maximum. By using polar cap models we are able to derive maximum polar cap sizes and acceptable viewing geometries. The inferred sizes of the caps turn out to be more compatible with a scenario in which the neutron star is heated by accretion, and place limits on the magnetic field strength. The hardness ratio modulation can then be explained in terms of energy-dependent beaming effects, and this constrains the acceptable models of the emerging radiation to cases in which softer photons are more strongly beamed than harder photons. An alternative explanation in terms of spatially variable absorption co-rotating in the magnetosphere is also discussed.
\end{abstract}

Key words. X-rays: stars - stars: neutron - stars: magnetic fields - individual: RX J0720.4-3125

\section{Introduction}

From its high ratio of X-ray to optical flux, soft X-ray spectrum, $8.4 \mathrm{~s}$ X-ray period and location in the Galactic plane, Haberl et al. (1997) proposed that the ROSAT source RX J0720.4-3125 is an isolated neutron star (NS). A probable optical counterpart has been identified (Motch \& Haberl 1998; Kulkarni \& van Kerkwijk 1998). Paerels et al. (2001, Paper I) present spectra of RX J0720.4-3125 using XMM-Newton, and find that there is no evidence for absorption lines and edges in the X-ray spectrum. They are able to refine the ROSAT spectral fits using blackbody spectra of $86.2 \pm 0.3 \mathrm{eV}$ absorbed by a cold absorber of $6.0 \pm 0.410^{19} \mathrm{~cm}^{-2}$.

Paper I mainly addressed the spectral characteristics and the behaviour of RX J0720.4-3125 averaged over the $8.4 \mathrm{~s}$ spin period. In this paper we examine the implications of the phase-resolved data.

\section{Observations}

Observations of RX J0720.4-3125 were made using XMMNewton (Jansen et al. 2001) on 2000 May 13 (orbit 78).

Send offprint requests to: M. Cropper

* Based on observations obtained with XMM-Newton, an ESA science mission with instruments and contributions directly funded by ESA Member States and the USA (NASA).
For the phase resolved data we have concentrated on the EPIC-PN data, as the EPIC-MOS provides insufficient time resolution in the modes selected for the observation. The raw data were processed using the version of the XMM-Newton Science Analysis System (Watson et al. 2001) released on 2000 July 12.

The particle background was significantly higher at the end of the observation, so those data were removed from the analysis, leaving a total observing time of $49 \mathrm{ks}$. Light curves were extracted from the EPIC-PN camera using an aperture $\sim 30^{\prime \prime}$ in radius centered on RX J0720.4-3125, chosen so that the aperture did not cover more than one CCD. This radius encompasses $\sim 90 \%$ of the integrated PSF (Aschenbach et al. 2000). We accumulated counts over the energy ranges $0.1-0.4,0.4-0.8$ and $0.8-1.2 \mathrm{keV}$ and phase-averaged them over 42 phase intervals on the $8.391 \mathrm{~s}$ period of Haberl et al. (1997). The spin profile in the band $0.1-1.2 \mathrm{keV}$ is shown in Fig. 1, together with the hardness ratio (soft/medium) variation and the best fit discussed in Sect. 4.

\subsection{Phase-folded X-ray light curves}

The general shape of the phased intensity curve is similar to that in Haberl et al. (1997) and is described in Paper I. It appears approximately symmetrical and sinusoidal with an amplitude $\sim 15 \%$. The hardness ratio is also seen to 


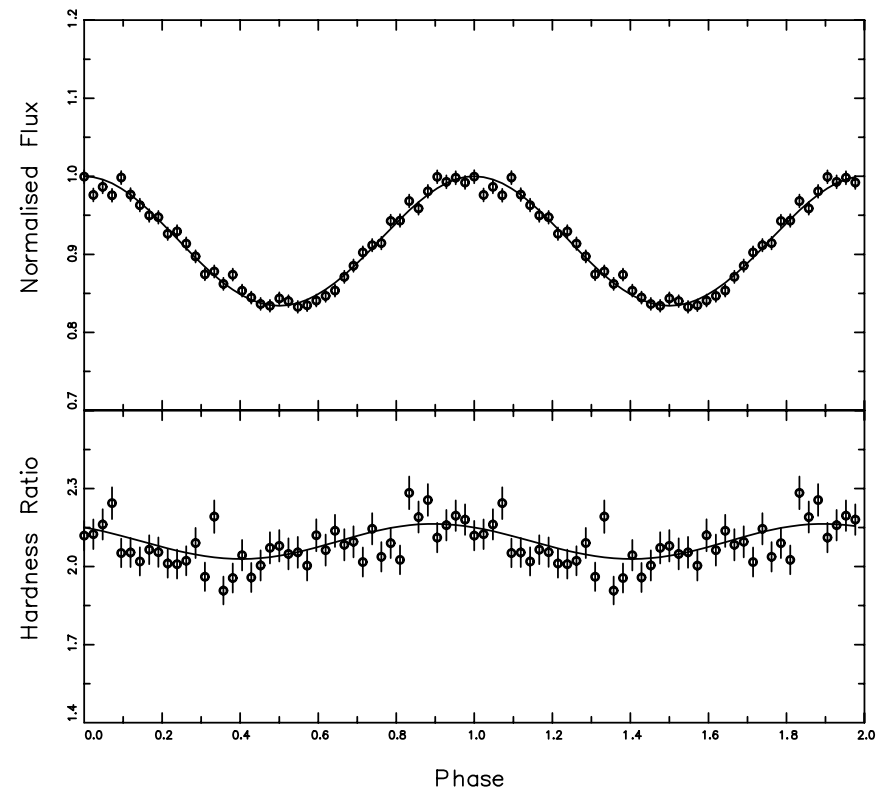

Fig. 1. The total flux (top) from EPIC-PN phase averaged on the $8.391 \mathrm{~s}$ period of Haberl et al. (1997) with the $(0.1$ to 0.4$) /(0.4$ to 0.8$) \mathrm{keV}$ hardness ratio (bottom). The flux is normalised to the model flux at phase 0.0. Also shown is the best fit to the data solid polar cap model with beaming and temperature variation (see Sect. 4 for details)

vary: it is softest around flux maximum and the amplitude is smaller at $\sim 10 \%$. The phasing of the hardness ratio curve is slightly but significantly earlier than the intensity curve. A cross-correlation of the two curves indicates that the phase difference is -0.048 .

The most likely source of the flux variation is the changing visibility of the heated magnetic polar caps (Haberl et al. 1997) with the rotation of the NS. Assuming symmetry, it is possible that the rotation period is twice that deduced by Haberl et al. (1997), depending on whether one cap or two are seen each rotation. A Fourier analysis excludes any harmonic or subharmonic to the level of $\sim 10 \%$ of the $8.391 \mathrm{~s}$ period but it is possible that odd-even effects may be present at lower levels. We have therefore also folded the data on a $16.782 \mathrm{~s}$ period. This gives rise to a lightcurve with two peaks per spin period with a small difference in amplitude. In this case the hardness ratio is also found to show two peaks per spin period.

\subsection{Phase resolved spectra}

We have selected data between phases 0.85-0.05 and 0.150.35 to examine more closely the change in spectral parameters in the "soft" and "hard" phase, i.e. at the maximum and minimum of the lightcurve, respectively. Spectra were background corrected using the same CCD on which the source was detected. In our fits we used the response file epn_fs20_sY9_thin.rmf produced by one of us (FH, version 2000 July) and we selected only single pixel events.

As in Paper I we have used a simple absorbed black body model in XSPEC. The spectral fit suggests that the majority of the variation is caused by a change in

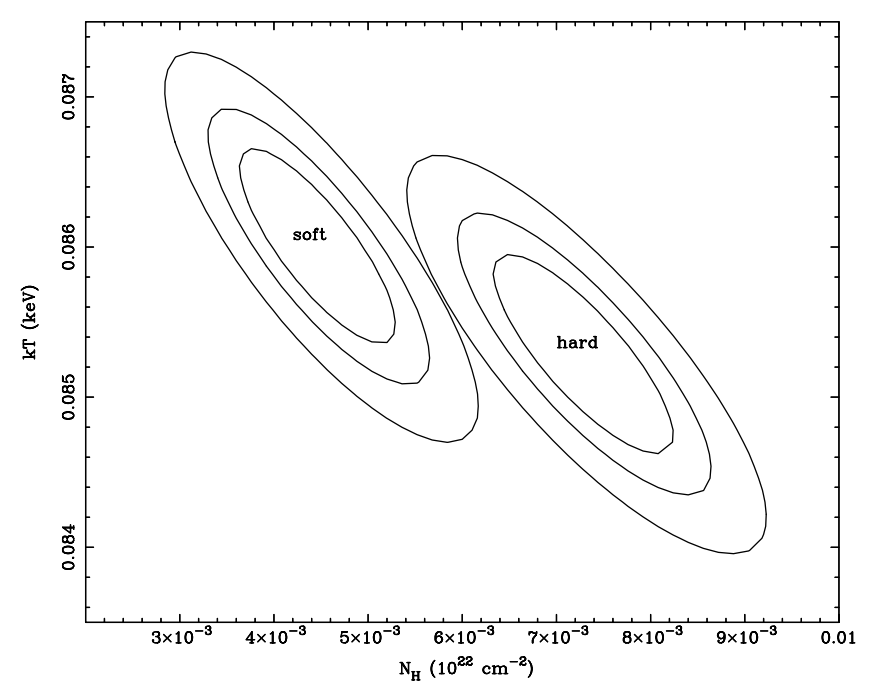

Fig. 2. Confidence contours in the temperature-absorbing column plane for the phases at the extremes of the hardness ratio variation in Fig. 1. Contours are for $68 \%, 90 \%$ and $99 \%$ confidence level

absorption, rather than a change in temperature (Fig. 2). The fits are consistent with a temperature of $85 \mathrm{eV}$, with the measured temperature difference between flux maximum and minimum of less than $1 \mathrm{eV}$. On the other hand, the best fit of the "hard" phase requires a doubling in absorption from $410^{19} \mathrm{~cm}^{-2}$ to $810^{19} \mathrm{~cm}^{-2}$. These parameters are consistent with the phase-averaged values from RGS data derived in Paper I. While residual uncertainties in the EPIC-PN calibration at these soft energies may introduce some uncertainties into the absolute values of the derived absorptions (see Paper I), it is unlikely that the relative change can be discounted.

\section{Analysis of the light curve}

\subsection{Polar cap models}

The high $S / N$ ratio of the X-ray pulsation in the $X M M$ Newton data provides the opportunity for a more detailed modelling than was possible using the ROSAT data. In this section, we first present the best fit of the light curve only, re-adapting the formalism originally derived by Pechenick et al. (1983). Assuming that the intensity variation is due to the changing visibility of heated circular magnetic polecaps and including gravity effects, those authors were able to derive a semi-analytical expression for the observed brightness, in terms of elliptic integrals. Their final result depends on four variables: the angular radius of the polecap $\alpha$, the angle between the dipole and rotation axes $\beta$, the angle between the line of sight and the rotation axis $\gamma$, and $R / 2 M$, where $M$ and $R$ are the mass and radius of the NS $(G=c=1)$. In addition, a prescription for the beaming function, $f(\delta)$, and a phase angle are required to complete the viewing angle calculation. The equations are symmetric for $\beta$ and $\gamma$ and $R / 2 M$ 
Table 1. The maximum angular size of the polar caps for different beaming prescriptions and compactness parameters

\begin{tabular}{crc}
\hline$f(\delta)$ & $R / 2 M$ & $\alpha_{\max }$ \\
\hline 1 & 4 & $53^{\circ}$ \\
& 3 & $44^{\circ}$ \\
$\cos \delta$ & 2.5 & $26^{\circ}$ \\
& 4 & $63^{\circ}$ \\
& 3 & $61^{\circ}$ \\
$\exp (-\delta)$ & 2.5 & $59^{\circ}$ \\
& 4 & $65^{\circ}$ \\
& 3 & $63^{\circ}$ \\
& 2.5 & $61^{\circ}$ \\
\hline
\end{tabular}

ranges between 2.5 and 4.0 for standard NS masses and equations of state.

Using this formalism, we have calculated model light curves for a range of parameter values. We have then used a Levenberg-Marquardt algorithm to fit the phased data by least squares. In order to maximise the precision of the fit we have used the spectral range of 0.1 to $1.2 \mathrm{keV}$ and we have weighted the fit using the reciprocal of the squares of the uncertainties.

\subsection{Fit to the models}

In the case of isotropic emission (no beaming, $f(\delta)=1$ ) the best fit to the data occurs for a polar cap radius of $\alpha=35^{\circ}$, dipole and viewing angles of $\beta=9.3^{\circ}$ and $\gamma=$ $42.7^{\circ}$, and $R / 2 M=4.2$. The quality of the fit is good, with $\chi_{\nu}^{2}=0.96$, and for these values the cap at the other pole is at most barely visible. The values of $\alpha$ and $\beta$ are the most sensitive to the angular distribution of the radiation field: when we change the beaming prescription by adopting a "pencil" model $(f(\delta)=\cos \delta)$, the best fit occurs for $\alpha=12.4, \beta=4.2, \gamma=41.3$ and $R / M=4.2\left(\chi_{\nu}^{2}=1.14\right)$.

However, the quasi-sinusoidal nature of the phased intensity data has the result that the phase space is degenerate for a wide range of input parameters. We have therefore taken the approach of fixing the cap radius and $R / 2 M$ and exploring the $(\beta, \gamma)$ plane for good fits. In the case of isotropic emission we have found that, for most cap radii and for $2.5 \leq R / 2 M \leq 4.0$, the acceptable parameter space is concentrated in a hyperbola described approximately by $\beta \sim C / \gamma$, where $C \sim 400$ for angles in degrees (see Fig. 3). As the cap size increases the best fit moves from the center of the arc where $\beta=\gamma$ to $(\beta, \gamma) \sim(10,48)$, and the constant $C$ increases slightly.

A physically more important result is that, for a given value of $R / 2 M$, the fits to the data constrain the maximum angular radius of the cap. By increasing $\alpha$, the $\chi_{\nu}^{2}$ of the best fit in $(\beta, \gamma)$ shows a sharp increase beyond a threshold value. We can therefore identify the maximum polecap radius beyond which a best fit can be rejected at the $90 \%$ confidence level $\left(\chi_{\nu}^{2}=4.61\right)$. Results are summarized in Table 1 for isotropic emission, a pencil model and a very extreme case in which the angular distribution of radiation decays exponentially with $\delta$. Smaller $R / 2 M$ require smaller maximum polecap radii because of

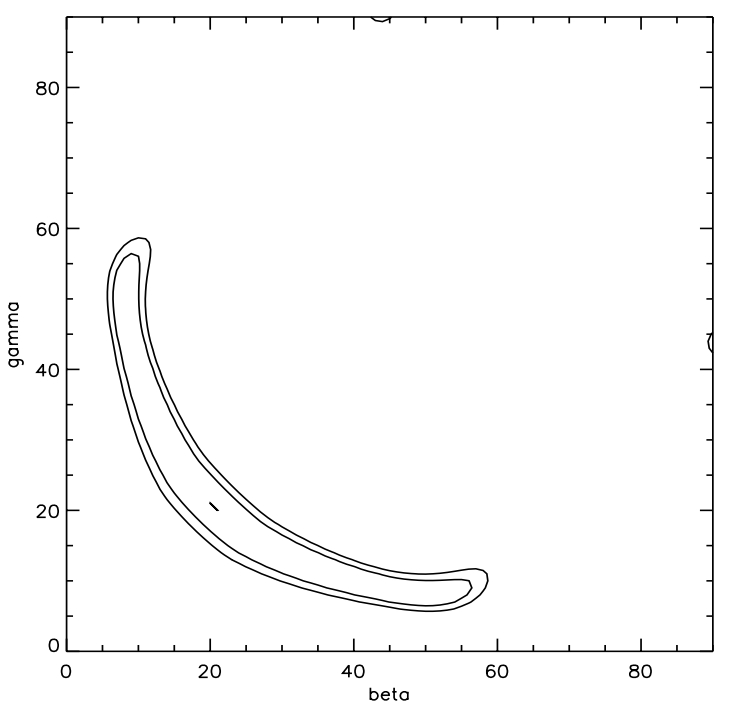

Fig. 3. The $\chi_{\nu}^{2}$ over the $(\beta, \gamma)$ plane for a cap angular radius $\alpha=10^{\circ}$ and $R / 2 M=3.0$. Here $f(\delta)=1$. Contours are for $\chi_{\nu}^{2}=1.0$ and then the $68 \%$ and $90 \%$ confidence intervals. The arc-shaped region and the small semicircular regions near the axes correspond to periods of $8.391 \mathrm{~s}$ and $16.782 \mathrm{~s}$, respectively

the increasing bending of the light ray trajectories from stars with large compactness parameter. In the presence of strong beaming effects, larger caps are allowed, but, at least when using simple, non energy-dependent beaming prescriptions, a polecap larger than $\sim 60^{\circ}-65^{\circ}$ can be rejected at a confidence level of $90 \%$.

\subsection{Constraints on $B$ from the size of the polar caps}

Independently on the interpretation of RX J0720.4-3125 as a cooling or an accreting NS, the existence of an upper limit for the cap size requires the NS to be magnetised.

A cooling NS interpretation implies magnetic fields much larger than in the accreting case. Such a high field, typically $10^{12}-10^{14} \mathrm{G}$, yields temperature variations with the magnetic colatitude, $\chi$, and modifies the emergent spectrum as function of viewing angle (Greenstein \& Hartke 1983; Page 1995). A simple expression for the surface temperature profile is (Possenti et al. 1996):

$T_{\text {surf }}=\frac{T_{\text {eff }}}{[1-0.47(1-K)]^{1 / 4}}\left[\frac{K+(4-K) \cos ^{2} \chi}{1+3 \cos ^{2} \chi}\right]^{1 / 4}$

where $K=k_{\perp} / k_{\|}$is the ratio of the coefficients of thermal conductivity orthogonal and parallel to the field: $K \sim 10^{-2}-10^{-4}$ for $B \sim 10^{11}-10^{13} \mathrm{G}$. Not even the maximum values of the polar cap size derived in Sect. 3.2 for strong beaming are fully compatible with this kind of interpretation: the temperature gradient given by equation (1) is so smooth that, even in the (unrealistic) limit case $K=0$ the associated blackbody luminosity drops only by a factor $1 / 2$ for $\alpha \sim 60^{\circ}$ and by an order of magnitude for $\alpha \sim 77^{\circ}$. Both the emergent spectrum and the degree of modulation of the signal are not strongly 
dependent on $K$. Despite the real angular distribution of the specific intensity being unknown and the fact that a proper modelling of the relative brightness should include the temperature profile, it seems difficult to explain the (relatively) small hot spot inferred from our calculations in terms of a surface temperature variation during the NS cooling phase.

If the polar caps are heated by accretion, the incoming material is channeled to the poles only by those field lines which have passed the Alfvèn radius. In the case of an aligned, dipolar $B$-field this gives $r_{\text {cap }} \sim R^{3 / 2} / r_{\mathrm{A}}^{1 / 2}$, where $r_{\text {cap }}, r_{\mathrm{A}}$ are the cap and the Alfvèn radius, respectively (see e.g. Eq. (9) in Treves et al. 2000). Thus, the previous values of the maximum polecap radii translate for the case of isotropic emission to $B>\left(510^{5}, 610^{4}, 210^{4}\right) \dot{M}_{11}^{1 / 2} \mathrm{G}$, where $\dot{M}_{11}=\dot{M} /\left(10^{11} \mathrm{~g} \mathrm{~s}^{-1}\right)$.

The minimum polecap radius is not constrained from the data: we find that good fits can be obtained even for $\alpha=1^{\circ}$ for all $2.5 \leq R / 2 M \leq 4.0$. In the framework of the accreting scenario, the existence of an upper limit $B \sim 10^{10} \mathrm{G}$ can be used to constrain the minimum $\alpha$ : for larger fields, in fact, accretion will be completely hindered. This gives a minimum $\alpha \sim 1^{\circ}$, which is indeed consistent with the data. Such low field strengths may provide one of the first indication that a significant field decay had occurred in an isolated NS, or, as it has been also suggested, may indicate that this object is the outcome of the common envelope evolution of a binary system (see Treves et al. 2000 and references therein; Wang 1997).

\section{Simultaneous analysis of light curve and hardness ratio variation}

The Pechenick et al. (1983) formalism assumes emission from a uniform temperature polecap, and it does not predict any hardness ratio changes through the spin cycle. However, zones of different temperatures can be used in conjunction with the spectral response of EPIC-PN and a spectral model for the emission to calculate the observed fluxes and hardness ratios. We use a blackbody to simulate the thermal emission as this provides a good approximation to the flux distribution of more detailed atmospheric models (at least in the X-ray band, see Zavlin et al. 1996; Zane et al. 2000). However the angular distribution of the emerging radiation in these models show a variety of beaming profiles, depending on chemical composition and magnetic field, which must be accounted for separately in the modelling, as implemented in Sect. 3.

The two main observational features are the simultaneous variations of hardness ratio and luminosity and their shift in phase. In addition, the spectral fit at the minimum of the lightcurve implies a larger absorption. By working on the premise of minimizing the number of additional assumptions, we investigated the parameter space using different simple models for the emission. We allow for a temperature variation inside the caps, for beaming effects, for variations in absorption and for asymmetric conditions
Table 2. Summary of acceptable models

\begin{tabular}{|c|c|c|c|c|c|c|}
\hline \multirow[b]{2}{*}{ Model } & \multicolumn{3}{|c|}{$8.391 \mathrm{~s}$ period } & \multicolumn{3}{|c|}{16.782 s period } \\
\hline & \begin{tabular}{|c|}
2 -pole \\
$\alpha<35^{\circ}$
\end{tabular} & $\begin{array}{c}2 \text {-pole } \\
\alpha>35^{\circ}\end{array}$ & 1-pole & $\begin{array}{c}2 \text {-pole } \\
\alpha<35^{\circ}\end{array}$ & $\begin{array}{c}2 \text {-pole } \\
\alpha>35^{\circ}\end{array}$ & 1-pole \\
\hline $\begin{array}{l}2 \text { polar caps at dif- } \\
\text { ferent temperatures }\end{array}$ & $x^{\mathrm{A}}$ & $x^{F}$ & $x^{\mathrm{C}}$ & $x^{D}$ & $x^{B}$ & $x^{\mathrm{B}}$ \\
\hline $\begin{array}{lr}2 \text { polar } & \text { caps with } \\
\text { different } & \text { absorp- } \\
\text { tions } & \\
\end{array}$ & $x^{A}$ & $x^{\mathrm{F}}$ & $x^{\mathrm{C}}$ & $x^{D}$ & $x^{B}$ & $x^{\mathrm{B}}$ \\
\hline $\begin{array}{l}2 \text { equal polar caps } \\
\text { with gradient in ab- } \\
\text { sorption }\end{array}$ & $x^{A}$ & O & 0 & O & $x^{B}$ & $x^{B}$ \\
\hline $\begin{array}{l}2 \text { equal polar caps } \\
\text { with gradient in } \\
\text { temperature }\end{array}$ & $x^{A}$ & $x^{\mathrm{E}}$ & $x^{\mathrm{E}}$ & $x^{\mathrm{E}}$ & $x^{B}$ & $x^{\mathrm{B}}$ \\
\hline $\begin{array}{l}2 \text { equal polar caps } \\
\text { with beaming }\end{array}$ & $x^{A}$ & $\bullet$ & $\bullet$ & $\bullet$ & $x^{B}$ & $x^{B}$ \\
\hline
\end{tabular}

- Acceptable fits possible.

- Probably unacceptable on grounds of poor model fit and unphysicality of absorption model.

A Excluded on the grounds of not being consistent with the $8.391 \mathrm{~s}$ period.

B Excluded on the grounds of not being consistent with the $16.782 \mathrm{~s}$ period.

C Excluded on the grounds that it predicts no hardness ratio variation.

D Excluded on the grounds that the hardness ratio variation would be on a $16.782 \mathrm{~s}$ period rather than the $8.391 \mathrm{~s}$ observed.

E Excluded on the grounds that the temperature difference required to produce the hardness ratio changes is not consistent with the spectral fit.

F Excluded on the grounds of the shape of the hardness ratio variation.

in the two spots. Despite the temperature of the dominant thermal component not varying during a period, the presence of a second component with $60 \mathrm{eV}<T<90 \mathrm{eV}$ (or, equivalently, of a smooth gradient of $T$ ) is still acceptable on spectral basis.

Our findings are summarized in Table 2. With "1-pole" and "2-pole" we refer to models in which one or two polar caps become visible during the rotation. Quite interestingly, most models can be discarded since they do not allow adequate fits. This is denoted by an $x$ in the table, together with the reason for their exclusion. We find that limb darkening variations are the best candidate to give rise to the expected modulation. If the emission is isotropic, the fact that the hardness ratio is softest near the flux maximum can be fitted only if the temperature decreases toward the center of the cap; however, the spectral dependence is inconsistent with the observed spectrum. On the other hand, by using different prescriptions for $f(\delta)$, we can also allow for a more realistic temperature profile, increasing towards the centre. The observational features require a beaming in the softer component much stronger than at highest energies. If this is the case, the change in $N_{\mathrm{H}}$ found with the simple absorbed blackbody model in XSPEC should mimic artificially the soft excess/deficit at different phases: this is naturally predicted by this kind of angular distribution. Our best fit model, $\chi_{\nu}^{2}=1.23$, is shown in Fig. 1 . The fit to the intensity data is good, but is not optimised: because of the number of degrees of freedom (now including the choice of spectral parameters and phase offsets) a fit was sought manually to the intensity data which would be both sufficient in formal 
terms and predict adequately the hardness ratio variation in mean value, amplitude and phase. In this model, we allow for a cooler ring with $T=70 \mathrm{eV}$, around a central, hotter region of $T=90 \mathrm{eV}$; the phase offset between them is 0.113 . The value of the column density has been fixed at $610^{19} \mathrm{~cm}^{-2}$, to be consistent with Paper I. The angular sizes of the soft and hard components are fitted by $\alpha_{\text {hot }}=33^{\circ}, \alpha_{\text {cold }}=38^{\circ}$, while the other parameters are $(\beta, \gamma, R / 2 M)=\left(5.3^{\circ}, 43.5^{\circ}, 4.2\right)$. The angular dependence of the emerging radiation is assumed to change from a pencil model, $f(\delta)=\cos \delta$, at low temperatures to isotropic emission, $f(\delta)=1$, at high temperatures. No reasonable fit can be found when the beaming is stronger at high energies, unless we require the polar cap to be colder at the center.

The inferred behaviour of the degree of anisotropy is contrary to what is physically expected at the simplest level in both cooling and accretion atmospheres. In an atmosphere in LTE where the main radiative process is bremsstrahlung, the opacity decreases with the photon energy. This, in turn, implies the anisotropy in the angular distribution being smaller at low frequencies (since these photons escape from the outermost atmospheric layers) and increases with the energy when photons decouple in the deepest, hottest layers. Moreover, as pointed out by Zavlin et al. (1996) for pure $\mathrm{H}$ cooling models, the maximum of the intensity spectrum shifts towards lower energies with increasing $\delta$, contrary to the hardness ratio variations. In order to match the hardness ratio variation, the energy-dependence of the degree of anisotropy must be reversed. For hydrogen atmospheres, that may happen only in the very high energy part of the spectrum, were the angular dependence tends to match the AmbartsumianChandrasekhar function for scattering dominated propagation (see Fig. 8 in Zavlin et al. 1996).

The observed change in $N_{\mathrm{H}}$ may be real (see Paper I), but, at least within our simple assumptions, models with different levels of absorption do not produce acceptable fits. Moreover, a variation in absorption over the spin cycle require the absorbing material to be close to the NS, probably co-rotating in the magnetosphere. In this case the light cylinder limits the maximum distance to the absorber to $R_{\max } \sim 410^{10} \mathrm{~cm}$. On the other hand, in order for the material to be substantially neutral, the ionisation parameter $\xi=L / n R^{2}$ must be less than 1 . This in turn requires a particle density $n>10^{10} \mathrm{~cm}^{-3}$, for $R<R_{\max }$ and a luminosity of $10^{31} \mathrm{erg} \mathrm{s}^{-1}$ (Haberl et al. 1997). A similar result can be obtained from the inferred increase in absorption of $410^{19} \mathrm{~cm}^{-2}$. For this to occur within $R_{\max }$ then $n>10^{9} \mathrm{~cm}^{-3}$. For comparison, typical densities of the interstellar medium are $n \sim 1 \mathrm{~cm}^{-3}$. The implication is that a reservoir of accreting material is held in the magnetosphere, in regions where gravitational, centrifugal and magnetic forces balance and confine it. These densities may be considered uncomfortably high, although we are unable to exclude them on current knowledge. We return to this in Sect. 5 .

\section{Conclusions}

By exploiting the superior quality of the data obtained from XMM-Newton, we have been able to present a detailed analysis of the spin pulse profile of RX J0720.43125. We have modelled these data by using polar cap models based on the Pechenick et al. (1983) formulation. The smooth nature of the data does not allow a unique conclusion about the best fit values to be reached, but nevertheless we are able to exclude a large volume of the parameter space and to derive an upper limit for the size of the emitting region. The derived values are at best marginally compatible with an interpretation in terms of surface temperature variation during the cooling phase of a middle-age NS. An accretor model is more satisfactory in accounting for the intensity plus hardness-ratio variations. Accreting NS appear to be relatively rare objects, as recently found by Popov et al. (2000) from a statistical evolutionary analysis. RX J0720.4-3125 may therefore be more important than perhaps initially appreciated.

We have investigated a number of possible explanations for the intensity and spectral variations over the spin period. The long period of RX J0720.4-3125 is somewhat unusual and hints at a possible evolutionary link between $\operatorname{dim}$ NS, soft gamma-ray repeaters (SGRs) and anomalous X-ray pulsars (AXPs) (Haberl et al. 1997; Alpar 2000), whereby isolated NS in the propellor phase are the progenitors of AXPs and SGRs. If the hardness ratio variation is indeed caused by absorption, then the large amount of material close by to the neutron star may be explained in terms of matter being propelled outwards. However the luminosity in this phase is caused by energy dissipation in the neutron star, and we have already found (Sect. 3.3) this requires polar cap sizes which are uncomfortably large. Moreover, such an evolutionary link would produce significantly larger numbers of AXPs and SGRs (Mereghetti, private communication).

The most plausible interpretation is that the observed modulation is produced by beaming effects in the emerging radiation. The angular distribution of the emerging radiation is still a matter for further investigation, and is extremely sensitive to details in the radiative computations and to a number of assumptions about chemical composition and magnetic field (see e.g. Zavlin et al. 1996; Rajagopal et al. 1997). Our modelling provides observational inputs for these investigations by suggesting that the softer X-rays from the heated cap are more strongly beamed than the harder X-rays.

Acknowledgements. We are grateful to R. Turolla and A. Possenti for several very helpful discussions, and to the referee for constructive comments.

\section{References}

Alpar, M. A. 2000 [astro-ph/0005211]

Aschenbach, B., Briel, U., Haberl, F., et al. 2000, SPIE, 4012,

Paper 86 [astro-ph/0007256]

Greenstein, G., \& Hartke, G. J. 1983, ApJ, 271, 283 
Haberl, F., Motch, C., Buckley, D. A. H., Zickgraf, F.-J., \& Pietsch, W. 1997, A\&A, 326, 662

Kulkarni, S. R., \& van Kerkwijk, M. H. 1998, ApJL, 507, L49 Jansen, F., Lumb, D., Altieri, B., et al. 2001, A\&A, 365, L1 Motch, C., \& Haberl, F. 1998, A\&A, 333, L59

Paerels, F., Mori, K., Motch, C., et al. 2001, A\&A, 365, L298

Page, D. 1995, ApJ, 442, 273

Pechenick, K. R., Ftaclas, C., \& Cohen, J. M. 1983, ApJ, 274, 846

Popov, S. B., Colpi, M., Treves, A., et al. 2000, ApJ, 530, 896

Possenti, A., Mereghetti, S., \& Colpi, M. 1996, A\&A, 313, 565
Rajagopal, M., Romani, R. W., \& Miller, M. C. 1997, ApJ, 479,347

Treves, A., Turolla, R., Zane, S., \& Colpi, M. 2000, PASP, 112, 297

Wang, J. C. L., 1997, ApJL, 486, L119

Watson, M. G., Auguères, J.-L., Ballet, J., et al. 2001, A\&A, $365, \mathrm{~L} 51$

Zane, S., Turolla, R., \& Treves, A. 2000, ApJ, 537, 387

Zavlin, V. E., Pavlov, G. G., \& Shibanov, Yu. A. 1996, A\&A, 315,141 